\title{
Graph Connectivity Measures for Unsupervised Parameter Tuning of Graph-Based Sense Induction Systems
}

\author{
Ioannis Korkontzelos, Ioannis Klapaftis and Suresh Manandhar \\ Department of Computer Science \\ The University of York \\ Heslington, York, YO10 5NG, UK \\ \{johnkork, giannis, suresh\}@cs.york.ac.uk
}

\begin{abstract}
Word Sense Induction (WSI) is the task of identifying the different senses (uses) of a target word in a given text. This paper focuses on the unsupervised estimation of the free parameters of a graph-based WSI method, and explores the use of eight Graph Connectivity Measures (GCM) that assess the degree of connectivity in a graph. Given a target word and a set of parameters, GCM evaluate the connectivity of the produced clusters, which correspond to subgraphs of the initial (unclustered) graph. Each parameter setting is assigned a score according to one of the GCM and the highest scoring setting is then selected. Our evaluation on the nouns of SemEval-2007 WSI task (SWSI) shows that: (1) all GCM estimate a set of parameters which significantly outperform the worst performing parameter setting in both SWSI evaluation schemes, (2) all GCM estimate a set of parameters which outperform the Most Frequent Sense (MFS) baseline by a statistically significant amount in the supervised evaluation scheme, and (3) two of the measures estimate a set of parameters that performs closely to a set of parameters estimated in supervised manner.
\end{abstract}

\section{Introduction}

Using word senses instead of word forms is essential in many applications such as information retrieval (IR) and machine translation (MT) (Pantel and Lin, 2002). Word senses are a prerequisite for word sense disambiguation (WSD) algorithms. However, they are usually represented as a fixed-list of definitions of a manually constructed lexical database. The fixed-list of senses paradigm has several disadvantages. Firstly, lexical databases often contain general definitions and miss many domain specific senses (Agirre et al., 2001). Secondly, they suffer from the lack of explicit semantic and topical relations between concepts (Agirre et al., 2001). Thirdly, they often do not reflect the exact content of the context in which the target word appears (Veronis, 2004). WSI aims to overcome these limitations of handconstructed lexicons.

Most WSI systems are based on the vector-space model that represents each context of a target word as a vector of features (e.g. frequency of cooccurring words). Vectors are clustered and the resulting clusters are taken to represent the induced senses. Recently, graph-based methods have been employed to WSI (Dorow and Widdows, 2003; Veronis, 2004; Agirre and Soroa, 2007b).

Typically, graph-based approaches represent each word co-occurring with the target word, within a pre-specified window, as a vertex. Two vertices are connected via an edge if they co-occur in one or more contexts of the target word. This cooccurrence graph is then clustered employing different graph clustering algorithms to induce the senses. Each cluster (induced sense) consists of words expected to be topically related to the particular sense. As a result, graph-based approaches assume that each context word is related to one and only one sense of the target one.

Recently, Klapaftis and Manandhar (2008) argued that this assumption might not be always valid, since a context word may be related to more than one senses of the target one. As a result, they pro- 
posed the use of a graph-based model for WSI, in which each vertex of the graph corresponds to a collocation (word-pair) that co-occurs with the target word, while edges are drawn based on the cooccurrence frequency of their associated collocations. Clustering of this collocational graph would produce clusters, which consist of a set of collocations. The intuition is that the produced clusters will be less sense-conflating than those produced by other graph-based approaches, since collocations provide strong and consistent clues to the senses of a target word (Yarowsky, 1995).

The collocational graph-based approach as well as the majority of state-of-the-art WSI systems estimate their parameters either empirically or by employing supervised techniques. The SemEval-2007 WSI task (SWSI) participating systems UOY and $U B C-A S$ used labeled data for parameter estimation (Agirre and Soroa, 2007a), while the authors of $I 2 R$, $U P V \_S I$ and $U M N D 2$ have empirically chosen values for their parameters. This issue imposes limits on the unsupervised nature of these algorithms, as well as on their performance on different datasets.

More specifically, when applying an unsupervised WSI system on different datasets, one cannot be sure that the same set of parameters is appropriate for all datasets (Karakos et al., 2007). In most cases, a new parameter tuning might be necessary. Unsupervised estimation of free parameters may enhance the unsupervised nature of systems, making them applicable to any dataset, even if there are no tagged data available.

In this paper, we focus on estimating the free parameters of the collocational graph-based WSI method (Klapaftis and Manandhar, 2008) using eight graph connectivity measures (GCM). Given a parameter setting and the associated induced clustering solution, each induced cluster corresponds to a subgraph of the original unclustered graph. A graph connectivity measure $G C M_{i}$ scores each cluster by evaluating the degree of connectivity of its corresponding subgraph. Each clustering solution is then assigned the average of the scores of its clusters. Finally, the highest scoring solution is selected.

Our evaluation on the nouns of SWSI shows that GCM improve the worst performing parameter setting by large margins in both SWSI evaluation schemes, although they are below the best perform- ing parameter setting. Moreover, the evaluation in a WSD setting shows that all GCM estimate a set of parameters which are above the Most Frequent Sense (MFS) baseline by a statistically significant amount. Finally our results show that two of the measures, i.e. average degree and weighted average degree, estimate a set of parameters that performs closely to a set of parameters estimated in a supervised manner. All of these findings, suggest that GCM are able to identify useful differences regarding the quality of the induced clusters for different parameter combinations, in effect being useful for unsupervised parameter estimation.

\section{Collocational graphs for WSI}

Let $b c$, be the base corpus, which consists of paragraphs containing the target word $t w$. The aim is to induce the senses of $t w$ given $b c$ as the only input. Let $r c$ be a large reference corpus. In Klapaftis and Manandhar (2008) the British National Corpus ${ }^{1}$ is used as a reference corpus. The WSI algorithm consists of the following stages.

Corpus pre-processing The target of this stage is to filter the paragraphs of the base corpus, in order to keep the words which are topically (and possibly semantically) related to the target one. Initially, $t w$ is removed from $b c$ and both $b c$ and $r c$ are PoS-tagged. In the next step, only nouns are kept in the paragraphs of $b c$, since they are characterised by higher discriminative ability than verbs, adverbs or adjectives which may appear in a variety of different contexts. At the end of this pre-processing step, each paragraph of $b c$ and $r c$ is a list of lemmatized nouns (Klapaftis and Manandhar, 2008).

In the next step, the paragraphs of $b c$ are filtered by removing common nouns which are noisy; contextually not related to $t w$. Given a contextual word $c w$ that occurs in the paragraphs of $b c$, a log-likelihood ratio $\left(G^{2}\right)$ test is employed (Dunning, 1993), which checks if the distribution of $c w$ in $b c$ is similar to the distribution of $c w$ in $r c ; p(c w \mid b c)=$ $p(c w \mid r c)$ (null hypothesis). If this is true, $G^{2}$ has a small value. If this value is less than a pre-specified threshold (parameter $p_{1}$ ) the noun is removed from $b c$.

${ }^{1}$ The British National Corpus (BNC) (2001, version 2). Distributed by Oxford University Computing Services. 


\begin{tabular}{|l|l|}
\hline Target: cnn_nbc & Target: nbc_news \\
\hline nbc_tv & nbc_tv \\
\hline cnn_tv & soap_opera \\
\hline cnn_radio & nbc_show \\
\hline news_newscast & news_newscast \\
\hline radio_television & nbc_newshour \\
\hline cnn_headline & cnn_headline \\
\hline nbc_politics & radio_tv \\
\hline breaking_news & breaking_news \\
\hline
\end{tabular}

Table 1: Collocations connected to $c n n \_n b c$ and $n b c \_n e w s$

This process identifies nouns that are more indicative in $b c$ than in $r c$ and vice versa. However, in this setting we are not interested in nouns which have a distinctive frequency in $r c$. As a result, each $c w$ which has a relative frequency in $b c$ less than in $r c$ is filtered out. At the end of this stage, each paragraph of $b c$ is a list of nouns which are assumed to be contextually related to the target word $t w$.

Creating the initial collocational graph The target of this stage is to determine the related nouns, which will form the collocations, and the weight of each collocation. Klapaftis and Manandhar (2008) consider collocations of size 2, i.e. pairs of nouns.

For each paragraph of $b c$ of size $n$, collocations are identified by generating all the possible $\left(\begin{array}{c}c_{n} \\ 2\end{array}\right)$ combinations. The frequency of a collocation $c$ is the number of paragraphs in the whole SWSI corpus (27132 paragraphs), in which $c$ occurs.

Each collocation is assigned a weight, measuring the relative frequency of two nouns co-occurring. Let $f_{r e q} q_{i j}$ denote the number of paragraphs in which nouns $i$ and $j$ cooccur, and $f_{r e q}$ denote the number of paragraphs, where noun $j$ occurs. The conditional probability $p(i \mid j)$ is defined in equation 1 , and $p(j \mid i)$ is computed in a similar way. The weight of collocation $c_{i j}$ is the average of these conditional probabilities $w_{c_{i j}}=p(i \mid j)+p(j \mid i)$.

$$
p(i \mid j)=\frac{\text { freq }_{i j}}{\text { freq }_{j}}
$$

Finally, Klapaftis and Manandhar (2008) only extract collocations which have frequency (parameter $p_{2}$ ) and weight (parameter $p_{3}$ ) higher than prespecified thresholds. This filtering appears to compensate for inaccuracies in $G^{2}$, as well as for lowfrequency distant collocations that are ambiguous. Each weighted collocation is represented as a ver- tex. Two vertices share an edge, if they co-occur in one or more paragraphs of $b c$.

\section{Populating and weighing the collocational graph}

The constructed graph, $G$, is sparse, since the previous stage attempted to identify rare events, i.e. co-occurring collocations. To address this problem, Klapaftis and Manandhar (2008) apply a smoothing technique, similar to the one in Cimiano et al. (2005), extending the principle that $a$ word is characterised by the company it keeps (Firth, 1957) to collocations. The target is to discover new edges between vertices and to assign weights to all edges.

Each vertex $i$ (collocation $c_{i}$ ) is associated to a vector $V C_{i}$ containing its neighbouring vertices (collocations). Table 1 shows an example of two vertices, $c n n \_n b c$ and $n b c \_n e w s$, which are disconnected in $G$ of the target word network. The example was taken from Klapaftis and Manandhar (2008).

In the next step, the similarity between all vertex vectors $V C_{i}$ and $V C_{j}$ is calculated using the Jaccard coefficient, i.e. $J C\left(V C_{i}, V C_{j}\right)=\frac{\left|V C_{i} \cap V C_{j}\right|}{\left|V C_{i} \cup V C_{j}\right|}$. Two collocations $c_{i}$ and $c_{j}$ are mutually similar if $c_{i}$ is the most similar collocation to $c_{j}$ and vice versa.

Given that collocations $c_{i}$ and $c_{j}$ are mutually similar, an occurrence of a collocation $c_{k}$ with one of $c_{i}, c_{j}$ is also counted as an occurrence with the other collocation. For example in Table 1, if $c n n \_n b c$ and $n b c \_n e w s$ are mutually similar, then the zerofrequency event between $n b c \_n e w s$ and $c n n \_t v$ is set equal to the joint frequency between $c n n \_n b c$ and cnn_tv. Marginal frequencies of collocations are updated and the overall result is consequently a smoothing of relative frequencies.

The weight applied to each edge connecting vertices $i$ and $j$ (collocations $c_{i}$ and $c_{j}$ ) is the maximum of their conditional probabilities: $p(i \mid j)=\frac{\text { freq }_{i j}}{\text { freq }_{j}}$, where $\mathrm{freq}_{i}$ is the number of paragraphs collocation $c_{i}$ occurs. $p(j \mid i)$ is defined similarly.

Inducing senses and tagging In this final stage, the collocational graph is clustered to produced the senses (clusters) of the target word. The clustering method employed is Chinese Whispers (CW) (Biemann, 2006). CW is linear to the number of graph edges, while it offers the advantage that it does not require any input parameters, producing the clusters of a graph automatically. 


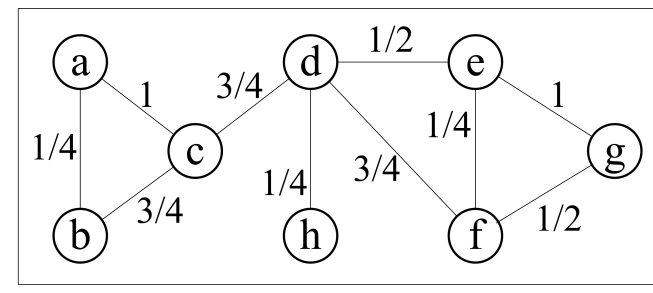

Figure 1: An example undirected weighted graph.

Initially, CW assigns all vertices to different classes. Each vertex $i$ is processed for a number of iterations and inherits the strongest class in its local neighbourhood (LN) in an update step. $\mathrm{LN}$ is defined as the set of vertices which share an edge with $i$. In each iteration for vertex $i$ : each class, $c l$, receives a score equal to the sum of the weights of edges $(i, j)$, where $j$ has been assigned to class $c l$. The maximum score determines the strongest class. In case of multiple strongest classes, one is chosen randomly. Classes are updated immediately, meaning that a vertex can inherit from its $\mathrm{LN}$ classes that were introduced in the same iteration.

Once CW has produced the clusters of a target word, each of the instances of $t w$ is tagged with one of the induced clusters. This process is similar to Word Sense Disambiguation (WSD) with the difference that the sense repository has been automatically produced. Particularly, given an instance of $t w$ in paragraph $p_{i}$ : each induced cluster $c l$ is assigned a score equal to the number of its collocations (i.e. pairs of words) occurring in $p_{i}$. We observe that the tagging method exploits the one sense per collocation property (Yarowsky, 1995), which means that WSD based on collocations is probably finer than WSD based on simple words, since ambiguity is reduced (Klapaftis and Manandhar, 2008).

\section{Unsupervised parameter tuning}

In this section we investigate unsupervised ways to address the issue of choosing parameter values. To this end, we employ a variety of GCM, which measure the relative importance of each vertex and assess the overall connectivity of the corresponding graph. These measures are average degree, cluster coefficient, graph entropy and edge density (Navigli and Lapata, 2007; Zesch and Gurevych, 2007).

GCM quantify the degree of connectivity of the produced clusters (subgraphs), which represent the senses (uses) of the target word for a given clustering solution (parameter setting). Higher values of GCM indicate subgraphs (clusters) of higher connectivity. Given a parameter setting, the induced clustering solution and a graph connectivity measure $G C M_{i}$, each induced cluster is assigned the resulting score of applying $G C M_{i}$ on the corresponding subgraph of the initial unclustered graph. Each clustering solution is assigned the average of the scores of its clusters (table 6), and the highest scoring one is selected.

For each measure, we have developed two versions, i.e. one which considers the edge weights in the subgraph, and a second which does not. In the following description the terms graph and subgraph are interchangeable.

Let $G=(V, E)$ be an undirected graph (induced sense), where $V$ is a set of vertices and $E=$ $\{(u, v): u, v \in V\}$ a set of edges connecting vertex pairs. Each edge is weighted by a positive weight, $W: w_{u v} \rightarrow[0, \infty)$. Figure 1 shows a small example to explain the computation of GCM. The graph consists of 8 vertices, $|V|=8$, and 10 edges, $|E|=10$. Edge weights appear on edges, e.g. $w_{a b}=\frac{1}{4}$.

Average Degree The degree ( $\mathrm{deg}$ ) of a vertex $u$ is the number of edges connected to $u$ :

$$
\operatorname{deg}(u)=|\{(u, v) \in E: v \in V\}|
$$

The average degree (AvgDeg) of a graph can be computed as:

$$
\operatorname{Avg} \operatorname{Deg}(G(V, E))=\frac{1}{|V|} \sum_{u \in V} \operatorname{deg}(u)
$$

The first row of table 2 shows the vertex degrees of the example graph (figure 1) and $\operatorname{Avg} \operatorname{Deg}(G)=$ $\frac{20}{8}=2.5$.

Edge weights can be integrated into the degree computation. Let mew be the maximum edge weight in the graph:

$$
\text { mew }=\max _{(u, v) \in E} w_{u v}
$$

Average Weighted Degree The weighted degree $\left(w \_d e g\right)$ of a vertex is defined as:

$$
w_{-} \operatorname{deg}(u)=\frac{1}{|V|} \sum_{(u, v) \in E} \frac{w_{u v}}{m e w}
$$




\begin{tabular}{|l|c|c|c|c|c|c|c|c|}
\cline { 2 - 9 } \multicolumn{1}{c|}{} & $\mathrm{a}$ & $\mathrm{b}$ & $\mathrm{c}$ & $\mathrm{d}$ & $\mathrm{e}$ & $\mathrm{f}$ & $\mathrm{g}$ & $\mathrm{h}$ \\
\hline $\operatorname{deg}(u)$ & 2 & 2 & 3 & 4 & 3 & 3 & 2 & 1 \\
\hline$w \operatorname{deg}(u)$ & $\frac{5}{4}$ & 1 & $\frac{5}{2}$ & $\frac{9}{4}$ & $\frac{7}{4}$ & $\frac{3}{2}$ & $\frac{3}{2}$ & $\frac{1}{4}$ \\
\hline$T_{u}$ & 1 & 1 & 1 & 1 & 1 & 2 & 1 & 0 \\
\hline$c c(u)$ & 1 & 1 & $\frac{1}{3}$ & $\frac{1}{6}$ & $\frac{1}{3}$ & $\frac{2}{3}$ & 1 & 0 \\
\hline$W T_{u}$ & $\frac{3}{4}$ & 1 & $\frac{1}{4}$ & $\frac{1}{4}$ & $\frac{1}{2}$ & $\frac{3}{2}$ & $\frac{1}{4}$ & 0 \\
\hline$w c c(u)$ & $\frac{3}{4}$ & 1 & $\frac{1}{12}$ & $\frac{1}{24}$ & $\frac{1}{6}$ & $\frac{1}{2}$ & $\frac{1}{4}$ & 0 \\
\hline$p(u)$ & $\frac{1}{10}$ & $\frac{1}{10}$ & $\frac{3}{20}$ & $\frac{1}{5}$ & $\frac{3}{20}$ & $\frac{3}{20}$ & $\frac{1}{10}$ & $\frac{1}{20}$ \\
\hline$e n(u) * 100$ & 33 & 33 & 41 & 46 & 41 & 41 & 33 & 22 \\
\hline$w p(u)$ & $\frac{1}{16}$ & $\frac{1}{20}$ & $\frac{1}{8}$ & $\frac{9}{80}$ & $\frac{7}{80}$ & $\frac{3}{40}$ & $\frac{3}{40}$ & $\frac{1}{80}$ \\
\hline$w e(u) * 100$ & 25 & 22 & 38 & 35 & 31 & 28 & 28 & 8 \\
\hline
\end{tabular}

Table 2: Computations of graph connectivity measures and relevant quantities on the example graph (figure 1).

Average weighted degree (AvgWDeg), similarly to AvgDeg, is averaged over all vertices of the graph. In the graph of figure 1 , mew $=1$. The second row of table 2 shows the weighted degrees of all vertices. $\operatorname{Avg} W \operatorname{Deg}(G)=\frac{48}{36} \simeq 1.33$.

Average Cluster Coefficient The cluster coefficient ( $c c$ ) of a vertex, $u$, is defined as:

$$
\begin{aligned}
c c(u) & =\frac{T_{u}}{T_{u}}=\sum_{(u, v) \in E} \sum_{\substack{v, x) \in E \\
x \neq u}} 1
\end{aligned}
$$

$T_{u}$ is the number of edges between the $k_{u}$ neighbours of $u$. Obviously $k_{u}=\operatorname{deg}(u) .2^{-1} k_{u}\left(k_{u}-1\right)$ would be the number of edges between the neighbours of $u$ if the graph they define was fully connected. Average cluster coefficient (AvgCC) is averaged over all vertices of the graph.

The computations of $T_{u}$ and $c c(u)$ on the example graph are shown in the third and fourth rows of table 2. Consequently, $\operatorname{Avg} C C(G)=\frac{9}{16}=0.5625$.

Average Weighted Cluster Coefficient Let $W T_{u}$ be the sum of edge weights between the neighbours of $u$ over mew. Weighted cluster coefficient (wcc) can be computed as:

$$
\begin{aligned}
& w c c(u)=\frac{W T_{u}}{2^{-1} k_{u}\left(k_{u}-1\right)} \\
& W T_{u}=\frac{1}{m e w} \sum_{(u, v) \in E} \sum_{\substack{v, x) \in E \\
x \neq u}} w_{v x}
\end{aligned}
$$

Average weighted cluster coefficient (AvgWCC) is averaged over all vertices of the graph. The computations of $W T_{u}$ and $w c c(u)$ on the example graph (figure 1) are shown in the fifth and sixth rows of table 2 and $\operatorname{Avg} W C C(G)=\frac{67}{8 * 24} \simeq 0.349$.

Graph Entropy Entropy measures the amount of information (alternatively the uncertainty) in a random variable. For a graph, high entropy indicates that many vertices are equally important and low entropy that only few vertices are relevant (Navigli and Lapata, 2007). The entropy (en) of a vertex $u$ can be defined as:

$$
e n(u)=-p(u) \log _{2} p(u)
$$

The probability of a vertex, $p(u)$, is determined by the degree distribution:

$$
p(u)=\left\{\frac{\operatorname{deg}(u)}{2|E|}\right\}_{u \in V}
$$

Graph entropy $(G E)$ is computed by summing all vertex entropies and normalising by $\log _{2}|V|$. The seventh and eighth row of table 2 show the computations of $p(u)$ and $e n(u)$ on the example graph, respectively. Thus, $G E \simeq 0.97$.

Weighted Graph Entropy Similarly to previous graph connectivity measures, the weighted entropy (wen) of a vertex $u$ is defined as:

$$
\begin{array}{r}
w e(u)=-w p(u) \log _{2} w p(u) \\
\text { where: } w p(u)=\left\{\frac{w_{-} \operatorname{deg}(u)}{2 * \text { mew } *|E|}\right\}_{u \in V}
\end{array}
$$

Weighted graph entropy $(G E)$ is computed by summing all vertex weighted entropies and normalising by $\log _{2}|V|$. The last two rows of table 2 show the computations of $w p(u)$ and $w e(u)$ on the example graph. Consequently, $W G E \simeq 0.73$.

Edge Density and Weighted Edge Density Edge density (ed) quantifies how many edges the graph has, as a ratio over the number of edges of a fully connected graph of the same size:

$$
A(V)=2\left(\begin{array}{c}
|V| \\
2
\end{array}\right)
$$


Edge density (ed) is a global graph connectivity measure; it refers to the whole graph and not a specific vertex. Edge density (ed) and weighted edge density (wed) can be defined as follows:

$$
\begin{aligned}
e d(G(V, E)) & =\frac{|E|}{A(V)} \\
\operatorname{wed}(G(V, E)) & =\frac{1}{A(V)} \sum_{(u, v) \in E} \frac{w_{u, v}}{m e w}
\end{aligned}
$$

In the graph of figure 1: $A(V)=2\left(\begin{array}{l}8 \\ 2\end{array}\right)=28$, $e d(G)=\frac{10}{28} \simeq 0.357, \sum \frac{w_{u, v}}{m e w}=6$ and $\operatorname{wed}(G)=$ $\frac{6}{28} \simeq 0.214$.

The use of the aforementioned GCM allows the estimation of a different parameter setting for each target word. Table 3 shows the parameters of the collocational graph-based WSI system (Klapaftis and Manandhar, 2008). These parameters affect how the collocational graph is constructed, and in effect the quality of the induced clusters.

\section{Evaluation}

\subsection{Experimental setting}

The collocational WSI approach was evaluated under the framework and corpus of SemEval-2007 WSI task (Agirre and Soroa, 2007a). The corpus consists of text of the Wall Street Journal corpus, and is hand-tagged with OntoNotes senses (Hovy et al., 2006). The evaluation focuses on all 35 nouns of SWSI. SWSI task employs two evaluation schemes. In unsupervised evaluation, the results are treated as clusters of contexts and gold standard (GS) senses as classes. In a perfect clustering solution, each induced cluster contains the same contexts as one of the classes (Homogeneity), and each class contains the same contexts as one of the clusters (Completeness). F-Score is used to assess the overall quality of clustering. Entropy and purity are also used, complementarily. F-Score is a better measure than entropy or purity, since F-Score measures both homogeneity and completeness, while entropy and purity measure only the former. In the second scheme, supervised evaluation, the training corpus is used to map the induced clusters to GS senses. The testing corpus is then used to measure WSD performance (Table 4, Sup. Recall).

The graph-based collocational WSI method is referred as Col-Sm (where "Col" stands for the "col-

\begin{tabular}{|l|l|l|}
\hline Parameter & Range & Value \\
\hline$G^{2}$ threshold & $5,10,15$ & $p_{1}=5$ \\
\hline Collocation frequency & $4,6,8,10$ & $p_{2}=8$ \\
\hline Collocation weight & $0.2,0.3,0.4$ & $p_{3}=0.2$ \\
\hline
\end{tabular}

Table 3: Parameters ranges and values in Klapaftis and Manandhar (2008)

locational WSI" approach and "Sm" for its version using "smoothing"). Col-Bl (where "Bl" stands for "baseline") refers to the same system without smoothing. The parameters of Col-Sm were originally estimated by cross-validation on the training set of SWSI. Out of 72 parameter combinations, the setting with the highest F-Score was chosen and applied to all 35 nouns of the test set. This is referred as Col-Sm-org (where "org" stands for "original") in Table 4 . Table 3 shows all values for each parameter, and the chosen values, under supervised parameter estimation $^{2}$. Col-Bl-org (Table 4) induces senses as Col-Sm-org does, but without smoothing.

In table 4, Col-Sm-w (respectively Col-Bl-w) refers to the evaluation of $\mathrm{Col}-\mathrm{Sm}(\mathrm{Col}-\mathrm{Bl})$, following the same technique for parameter estimation as in Klapaftis and Manandhar (2008) for each target word separately ("w" stands for "word"). Given that GCM are applied for each target word separately, these baselines will allow to see the performance of GCM compared to a supervised setting.

The 1clinst baseline assigns each instance to a distinct cluster, while the $1 \mathrm{c} 1 \mathrm{w}$ baseline groups all instances of a target word into a single cluster. $1 \mathrm{c} 1 \mathrm{w}$ is equivalent to MFS in this setting. The fifth column of table 4 shows the average number of clusters.

The SWSI participant systems $U O Y$ and $U B C-A S$ used labeled data for parameter estimation. The authors of $I 2 R, U P V \_S I$ and $U M N D 2$ have empirically chosen values for their parameters.

The next subsection presents the evaluation of GCM as well as the results of SWSI systems. Initially, we provide a brief discussion on the differences between the two evaluation schemes of SWSI that will allow for a better understanding of GCM performance.

\subsection{Analysis of results and discussion}

Evaluation of WSI methods is a difficult task. For instance, 1clinst (Table 4) achieves perfect purity

\footnotetext{
${ }^{2} \mathrm{CW}$ performed 200 iterations for all experiments, because it is not guaranteed to converge.
} 


\begin{tabular}{|l|cccc|l|}
\hline \multicolumn{1}{|c|}{ System } & \multicolumn{4}{|c|}{ Unsupervised Evaluation } & Sup. \\
& FSc. & Pur. & Ent. & \# Cl. & Recall \\
\hline Col-Sm-org & 78.0 & 88.6 & 31.0 & 5.9 & 86.4 \\
Col-Bl-org & 73.1 & 89.6 & 29.0 & 8.0 & 85.6 \\
Col-Sm-w & 80.9 & 88.0 & 32.5 & 4.3 & 85.5 \\
Col-Bl-w & 78.1 & 88.3 & 31.7 & 5.4 & 84.3 \\
UBC-AS & 80.8 & 83.6 & 43.5 & 1.6 & 80.7 \\
UPV_SI & 69.9 & 87.4 & 30.9 & 7.2 & 82.5 \\
I2R & 68.0 & 88.4 & 29.7 & 3.1 & 86.8 \\
UMND2 & 67.1 & 85.8 & 37.6 & 1.7 & 84.5 \\
UOY & 65.8 & 89.8 & 25.5 & 11.3 & 81.6 \\
1c1w-MFS & 80.7 & 82.4 & 46.3 & 1 & 80.9 \\
1c1inst & 6.6 & 100 & 0 & 73.1 & N/A \\
\hline
\end{tabular}

Table 4: Evaluation of WSI systems and baselines.

and entropy. However, F-Score of 1clinst is low, because the GS senses are spread among clusters, decreasing unsupervised recall. Supervised recall of lclinst is undefined, because each cluster tags only one instance. Hence, clusters tagging instances in the test corpus do not tag any instances in the train corpus and the mapping cannot be performed. $1 c 1 w$ achieves high F-Score due to the dominance of MFS in the testing corpus. However, its purity, entropy and supervised recall are much lower than other systems, because it only induces the dominant sense.

Clustering solutions that achieve high supervised recall do not necessarily achieve high F-Score, mainly because F-Score penalises systems for inducing more clusters than the corresponding GS classes, as 1cllinst does. Supervised evaluation seems to be more neutral regarding the number of clusters, since clusters are mapped into a weighted vector of senses. Thus, inducing a number of clusters similar to the number of senses is not a requirement for good results (Agirre and Soroa, 2007a). High supervised recall means high purity and entropy, as in $I 2 R$, but not vice versa, as in $U O Y$. UOY produces many clean clusters, however these are unreliably mapped to senses due to insufficient training data. On the contrary, $I 2 R$ produces a few clean clusters, which are mapped more reliably.

Comparing the performance of SWSI systems shows that none performs well in both evaluation settings, in effect being biased against one of the schemes. However, this is not the case for the collocational WSI method, which achieves a high performance in both evaluation settings.

Table 6 presents the results of applying the graph

\begin{tabular}{|c|c|c|c|c|c|c|}
\hline \multirow[t]{2}{*}{ System } & \multirow{2}{*}{$\begin{array}{l}\text { Bound } \\
\text { type }\end{array}$} & \multicolumn{4}{|c|}{ Unsupervised Evaluation } & \multirow{2}{*}{$\begin{array}{l}\text { Sup. } \\
\text { Recall }\end{array}$} \\
\hline & & FSc. & Pur. & Ent. & \# Cl. & \\
\hline Col-Sm & MaxR & 79.3 & 90.5 & 26.6 & 7.0 & 88.6 \\
\hline Col-Sm & $\operatorname{MinR}$ & 62.9 & 89.0 & 26.7 & 12.7 & 78.8 \\
\hline Col-Bl & MaxR & 72.9 & 91.8 & 23.2 & 9.6 & 88.7 \\
\hline Col-Bl & MinR & 57.5 & 89.0 & 26.4 & 14.4 & 76.2 \\
\hline Col-Sm & MaxF & 83.2 & 90.0 & 28.7 & 4.9 & 86.6 \\
\hline Col-Sm & $\operatorname{MinF}$ & 43.6 & 90.2 & 22.1 & 17.6 & 83.7 \\
\hline Col-Bl & MaxF & 81.1 & 90.0 & 28.7 & 5.3 & 81.8 \\
\hline Col-B1 & MinF & 34.1 & 90.5 & 20.5 & 20.4 & 81.5 \\
\hline
\end{tabular}

Table 5: Upper and lower performance bounds for systems $\mathrm{Col}-\mathrm{Sm}$ and $\mathrm{Col}-\mathrm{Bl}$.

connectivity measures of section 3 in order to choose the parameter values for the collocational WSI system, for each word separately. The evaluation is done both for $\mathrm{Col}-\mathrm{Sm}$ and $\mathrm{Col}-\mathrm{Bl}$ that use and ignore smoothing, respectively.

To evaluate the supervised recall performance using the graph connectivity measures, we computed both the upper and lower bounds of Col-Sm, i.e. the best and worst supervised recall, respectively (MaxR and MinR in table 5). In the former case, we selected the parameter combination per target word that performs best (Col-Sm, MaxR in table 5), which resulted in $88.6 \%$ supervised recall (F-Score: $79.3 \%$ ), while in the latter we selected the worst performing one, which resulted in $78.8 \%$ supervised recall (F-Score: $62.9 \%$ ). In table 6 we observe that the supervised recall of all measures is significantly lower than the upper bound. However, all measures perform significantly better than the lower bound (McNemar's test, confidence level: 95\%); the smallest difference is $4.9 \%$, in the case of weighted edge density. The picture is the same for $\mathrm{Col}-\mathrm{Bl}$.

In the same vein, we computed both the upper and lower bounds of $\mathrm{Col}$-Sm in terms of F-Score, $83.2 \%$ and $43.6 \%$, respectively (Col-Sm, MinF and MaxF in table 5). The performance of the system is lower than the upper bound, for all GCM. Despite that, we observe that all measures except edge density and weighted edge density outperform the lower bound by large margins.

The comparison of GCM performance against the lower and upper bounds of $\mathrm{Col}-\mathrm{Sm}$ and $\mathrm{Col}-\mathrm{Bl}$ shows that GCM are able to identify useful differences regarding the degree of connectivity of induced clusters, and in effect suggest parameter values that perform significantly better than the worst 


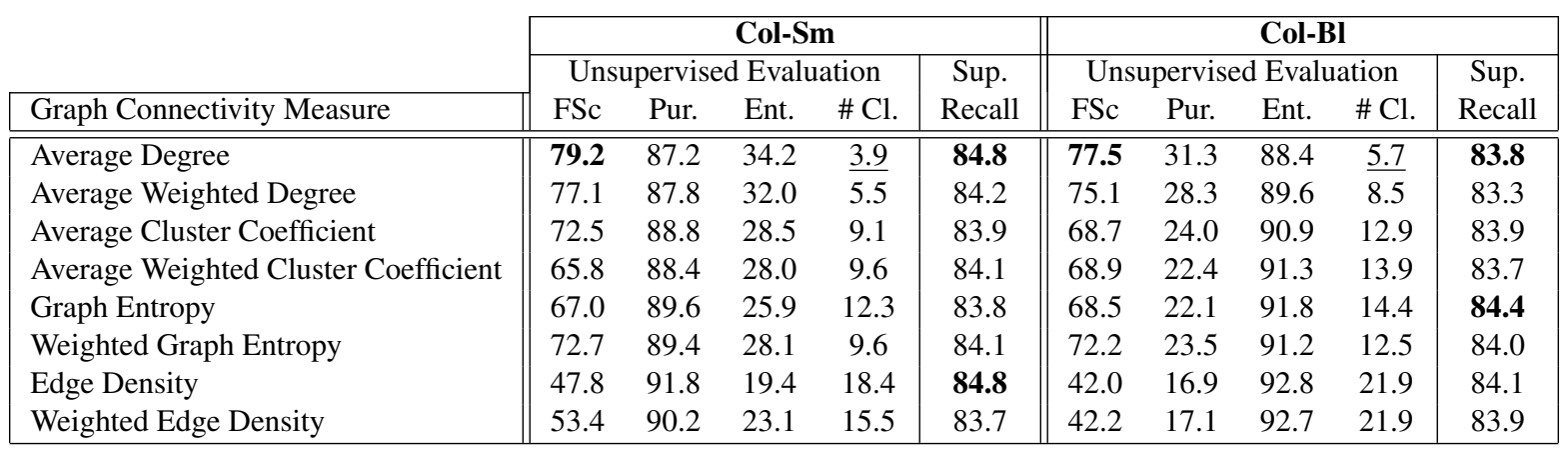

Table 6: Unsupervised \& supervised evaluation of the collocational WSI approach using graph connectivity measures.

case. However, they are all unable to approximate the upper bound for both evaluation schemes, which is also the case for the supervised estimation of parameters per target word (Col-Sm-w and Col-Bl-w).

In Table 6, we also observe that all measures achieve higher supervised recall scores than the MFS baseline. The increase is statistically significant (McNemar's test, confidence level: 95\%) in all cases. This result shows that irrespective of the number of clusters produced (low F-Score), GCM are able to estimate a set of parameters that provides clean clusters (low entropy), which when mapped to GS senses improve upon the most frequent heuristic, unlike the majority of unsupervised WSD systems.

Regarding the comparison between different GCM, we observe that average degree and weighted average degree for $\mathrm{Col}-\mathrm{Sm}$ (Col-Bl) perform closely to Col-Sm-w (Col-Bl-w) for both evaluation schemes. This is due to the fact that they produce a number of clusters similar to Col-Sm-w (Col-Bl-w), while at the same time their distributions of clusters over the target words' instances are also similar.

On the contrary, the remaining GCM tend to produce larger numbers of clusters compared to both Col-Sm-w (Col-Bl-w) and the GS, in effect being penalised by F-Score. As it has already been mentioned, supervised recall is less affected by a large number of clusters, which causes small differences among GCM.

Determining whether the weighted or unweighted version of GCM performs better depends on the GCM itself. Weighted graph entropy performs in all cases better than the unweighted version. For average cluster coefficient and edge density, we cannot extract a safe conclusion. Unweighted average degree performs better than the weighted version.

\section{Conclusion and future work}

In this paper, we explored the use of eight graph connectivity measures for unsupervised estimation of free parameters of a collocational graph-based WSI system. Given a parameter setting and the associated induced clustering solution, each cluster was scored according to the connectivity degree of its corresponding subgraph, as assessed by a particular graph connectivity measure. Each clustering solution was then assigned the average of its clusters' scores, and the highest scoring one was selected.

Evaluation on the nouns of SemEval-2007 WSI task (SWSI) showed that all eight graph connectivity measures choose parameters for which the corresponding performance of the system is significantly higher than the lower performance bound, for both the supervised and unsupervised evaluation scheme. Moreover, the selected parameters produce results which outperform the MFS baseline by a statistically significant amount in the supervised evaluation scheme. The best performing measures, average degree and weighted average degree, perform comparably well to the set of parameters chosen by a supervised parameter estimation. In general, graph connectivity measures can quantify significant differences regarding the degree of connectivity of induced clusters.

Future work focuses on further exploiting graph connectivity measures. Graph theoretic literature proposes a variety of measures capturing graph properties. Some of these measures might help in improving WSI performance, while at the same time keeping graph-based WSI systems totally unsupervised. 


\section{References}

Eneko Agirre and Aitor Soroa. 2007a. Semeval-2007 task 02: Evaluating word sense induction and discrimination systems. In Proceedings of the Fourth International Workshop on Semantic Evaluations (SemEval2007), pages 7-12, Prague, Czech Republic. Association for Computational Linguistics.

Eneko Agirre and Aitor Soroa. 2007b. Ubc-as: A graph based unsupervised system for induction and classification. In Proceedings of the Fourth International Workshop on Semantic Evaluations (SemEval-2007), pages 346-349, Prague, Czech Republic. Association for Computational Linguistics.

Eneko Agirre, Olatz Ansa, Eduard Hovy, and David Martinez. 2001. Enriching wordnet concepts with topic signatures, Sep.

Chris Biemann. 2006. Chinese whispers - an efficient graph clustering algorithm and its application to natural language processing problems. In Proceedings of TextGraphs: the Second Workshop on Graph Based Methods for Natural Language Processing, pages 7380, New York City, June. Association for Computational Linguistics.

Philipp Cimiano, Andreas Hotho, and Steffen Staab. 2005. Learning concept hierarchies from text corpora using formal concept analysis. Journal of Artificial Intelligence research, 24:305-339.

Beate Dorow and Dominic Widdows. 2003. Discovering corpusspecific word senses. In Proceedings 10th conference of the European chapter of the ACL, pages 79-82, Budapest, Hungary.

Ted E. Dunning. 1993. Accurate methods for the statistics of surprise and coincidence. Computational Linguistics, 19(1):61-74.

John R. Firth. 1957. A synopsis of linguistic theory, 1930-1955. Studies in Linguistic Analysis, pages 132.

Eduard Hovy, Mitchell Marcus, Martha Palmer, Lance Ramshaw, and Ralph Weischedel. 2006. Ontonotes: The 90\% solution. In Proceedings of the Human Language Technology Conference of the NAACL, Companion Volume: Short Papers, pages 57-60, New York City, USA. Association for Computational Linguistics.

Damianos Karakos, Jason Eisner, Sanjeev Khudanpur, and Carey Priebe. 2007. Cross-instance tuning of unsupervised document clustering algorithms. In Human Language Technologies 2007: The Conference of the North American Chapter of the Association for Computational Linguistics; Proceedings of the Main Conference, pages 252-259, Rochester, New York, April. Association for Computational Linguistics.

Ioannis P. Klapaftis and Suresh Manandhar. 2008. Word sense induction using graphs of collocations. In In
Proceedings of the 18th European Conference on Artificial Intelligence, (ECAI-2008), Patras, Greece.

R. Navigli and M. Lapata. 2007. Graph connectivity measures for unsupervised word sense disambiguation. In 20th International Joint Conference on Artificial Intelligence (IJCAI 2007), pages 1683-1688, Hyderabad, India, January.

Patrick Pantel and Dekang Lin. 2002. Discovering word senses from text. In KDD '02: Proceedings of the eighth ACM SIGKDD international conference on Knowledge discovery and data mining, pages 613619, New York, NY, USA. ACM Press.

Jean Veronis. 2004. Hyperlex: lexical cartography for information retrieval. Computer Speech \& Language, 18(3):223-252, July.

David Yarowsky. 1995. Unsupervised word sense disambiguation rivaling supervised methods. In Meeting of the Association for Computational Linguistics, pages 189-196.

Torsten Zesch and Iryna Gurevych. 2007. Analysis of the wikipedia category graph for NLP applications. In Proceedings of the Second Workshop on TextGraphs: Graph-Based Algorithms for Natural Language Processing, pages 1-8, Rochester, NY, USA. Association for Computational Linguistics. 